\title{
Vesicle Formation and General Phase Behavior in the Catanionic Mixture SDS-DDAB - Water. The Anionic-Rich Side
}

\author{
Eduardo F. Marques, ${ }^{*}, \dagger$ Oren Regev,$\dagger$ Ali Khan, ${ }^{\dagger}$ Maria da Graça Miguel,,$\star$ and \\ Björn Lindman ${ }^{\dagger}$
}

Physical Chemistry 1, Center for Chemistry and Chemical Engineering, P.O. Box 124, Lund University, Lund S-221 00, Sweden, and Departamento de Química, Universidade de Coimbra, 3049 Coimbra, Portugal

Received: December 1, 1997; In Final Form: June 9, 1998

\begin{abstract}
Catanionic mixtures are aqueous mixtures of oppositely charged surfactants which display novel phase behavior and interfacial properties in comparison with those of the individual surfactants. One phase behavior property is the ability of these systems to spontaneously form stable vesicles at high dilution. The phase behavior of the mixture sodium dodecyl sulfate (SDS) - didodecyldimethylammonium bromide (DDAB) in water has been studied in detail, and two regions of isotropic vesicular phases (anionic-rich and cationic-rich) were identified. Cryo-transmission electron microscopy allowed direct visualization of relatively small and polydisperse unilamellar vesicles on the SDS-rich side. Monitoring of the microstructure evolution from mixed micelles to vesicles as the surfactant mixing ratio is varied toward equimolarity was also obtained. Further information was provided by water self-diffusion measurements by pulsed field gradient spin-echo NMR. Water molecules can be in fast or slow exchange between the inside and outside of the vesicle with respect to the experimental time scale, depending on membrane permeability and vesicle size. For the SDSrich vesicles, a slow-diffusing component of very low molar fraction observed for the echo decays was traced down to very large vesicles in solution. Light microscopy confirmed the presence of vesicles of several microns in diameter. Thus, polydispersity seems to be an inherent feature of the system.
\end{abstract}

\section{Introduction}

Catanionic mixtures are pseudoternary aqueous mixtures of two oppositely charged surfactants which display interesting interfacial and phase behavior properties, determined by surfactant mixing ratio, total concentration, and molecular structure. ${ }^{1-3}$ At high surfactant concentration and above the Krafft boundary of the mixture, these systems show a variety of liquid crystalline phases, namely, lamellar and cubic phases, which are not present in the individual surfactant-water binary systems. ${ }^{4-6}$ At low concentration, typically below $5 \mathrm{wt} \%$, their behavior is dominated by phase separation, with the formation of highly insoluble precipitates (catanionic solid) at and around equimolarity. However, with an excess of one of the surfactants, the precipitate dissolves to yield solutions (and appropriate heterogeneous regions) containing self-assembled aggregates of different structures. These solutions have been known for a long time to possess important synergistic interfacial phenomena, such as the lowering of critical aggregation concentrations and enhanced surface activity, adsorption, and detergency, with relevance to application-oriented goals. Yet, it is the structural studies on these isotropic phases that have in recent years attracted considerable interest, mainly due to the fact that spontaneous formation of stable vesicles appears to be a general feature of these mixtures. . $^{3,7}$

A vesicle is a colloidal object, usually spherical, which consists of a self-folded amphiphilic bilayer enclosing a volume

* Author for correspondence. Tel.: 46462228148 . Fax: 4646224413. Email: eduardo.marques@fkem1.lu.se.

$\dagger$ Lund University.

$\div$ Universidade de Coimbra.

$\S$ Permanent address: Department of Chemical Engineering, Ben-Gurion University, P.O.Box 653, 84105 Beer-Sheva, Israel. of solvent secluded from the bulk. A vesicular solution always presents some degree of polydispersity, and the average vesicle diameter varies enormously between systems, from small unilamellar vesicles $(10-50 \mathrm{~nm})$ to large unilamellar $(50-500$ $\mathrm{nm}$ ), giant unilamellar (more than $0.5 \mu \mathrm{m}$ ) and multilamellar vesicles $(1-50 \mu \mathrm{m})$, also known as onions. The interest in vesicular phases is ubiquitous, ranging from fundamental areas within biochemistry and physical chemistry to application aspects in microreactor chemistry, pharmacology (drug delivery), medicine and cosmetics. ${ }^{8-10}$ Vesicles composed of single or mixed phospholipids and other water-insoluble surfactants can be readily prepared with different sizes and controlled polydispersity. This is done by treating mechanically (typically by ultrasonication), chemically, or by other elaborate methods the dilute lamellar dispersions (biphasic regions with a lamellar phase and excess solution) that these insoluble surfactants form in water. As such, the vesicular solutions are not thermodynamically stable-eventually they revert to the original equilibrium lamellar dispersion. ${ }^{9}$

On the other hand, catanionic vesicles have been shown to form spontaneously and reversibly and to remain stable for long periods of time, which strongly suggests that they are thermodynamically stable systems. Since the initial work by Hargreaves and Deamer ${ }^{11}$ and Kaler and co-workers, ${ }^{7,12-14}$ several reports have been presented addressing vesiculation of catanionic mixtures. ${ }^{15-19}$ Theoretical models accounting for the stability of these vesicles have also been proposed..$^{20-22}$ However, it is clear that further structural and phase behavior characterization of this type of vesicle-forming system is needed, so that a more fundamental understanding of vesiculation phenomena can be attained. The study of the kinetic aspects and the influence of preparation methods (if any) on the formation and size distribu- 
tion of vesicles is a central issue. The evolution of the aggregate structure as the surfactant mixing ratio is varied, namely, the micelle-to-vesicle transition, is also a topic of interest in catanionic mixtures. ${ }^{13,16,19,23}$ The fact that these aggregate structures often occur for very dilute solutions and often show high polydispersity in size and shape poses difficulties in terms of the availability of characterization techniques. Direct aggregate visualization by electron and light microscopy techniques has to be used in combination with data from more quantitative techniques such as NMR, light scattering, and fluorescence.

In previous work, we have reported the phase behavior for the catanionic system sodium dodecyl sulfate (SDS)-didodecyldimethylammonium bromide (DDAB) - water and mentioned the occurrence of vesicles in the very dilute region for both the anionic- and cationic-rich area, on the basis of particle sizing by light scattering. ${ }^{4}$ The area of existence of the vesicle solutions was not determined in detail. Recently, Kondo et al. have investigated the area of anionic-rich vesicles in this system and presented a preliminary view of the phase behavior and vesicle characterization. ${ }^{24}$ In this work, we confirm the occurrence of the two vesicle isotropic regions on the water-rich corner of this catanionic mixture and present a detailed portrayal of the phase behavior. The structural characterization of the vesicles in terms of size, polydispersity, and stability is given on the basis of direct structural imaging by microscopy (cryoTEM and light microscopy) and water NMR self-diffusion techniques.

DDAB is a water-insoluble, double-chained surfactant with a critical packing parameter, as defined by Israelachvili, ${ }^{25}$ close to unity. ${ }^{26}$ It forms bilayer structures in water: a concentrated and a swollen lamellar phase separated by a region of coexistence of the two phases ${ }^{27-29}$ and at high dilution a dispersion containing large vesicular assemblies. ${ }^{30,31}$ SDS, on the other hand, is a water-soluble, micelle-forming surfactant, with a packing parameter of about $1 / 3^{25}$ in dilute solution. Thus, the strong electrostatic headgroup interactions will be modulated by the packing constraints imposed by the geometry, in the dictation of the preferred aggregate structure for a given composition. In the present report, we address the phase behavior and microstructure of the anionic-rich side of the phase diagram; the cationic-rich side is dealt with in a complementary study.

\section{Experimental Section}

Materials and Sample Preparation. Specially pure SDS was purchased from BDH, England, and used without further purification, since the $\mathrm{cmc}$ determination by surface tension (du Noüy platinum ring method) yielded a value very close to 8 $\mathrm{mM}$, in agreement with the accepted value. DDAB of high purity from Tokyo Kasei, Japan, was used as obtained. The samples were mixed either by weight or by volume, depending on their composition. For samples with more than $2 \mathrm{wt} \%$ in total surfactant, the two solids were weighed and thoroughly mixed in water. For more dilute samples, the appropriate volumes of an SDS micellar solution and a DDAB pseudosolution, i.e., a carefully homogenized lamellar dispersion, were mixed. DDAB forms relatively viscous turbid dispersions, which phase-separate after days to weeks (when left undisturbed) into a solution with a flow birefringent layer at the top. However, if homogenized by gentle shaking for 1-3 days, the dispersion changes to a metastable solution, from which the required volume was pipetted out.

Phase Diagram Determination. After preparation, the samples were left for equilibration for several days prior to the examination of phase behavior. Inspection between crossed polaroids followed in order to search for birefringent phases. Samples belonging to the vesicular region were observed over the course of months in order to check for any phase separation phenomena, such as flocculation or crystal precipitation.

Light Microscopy. An Axioplan Universal light microscope from Carl Zeiss, equipped with differential interference contrast (DIC) lenses, was used. The main goal was to check for the presence of crystals or anisotropic liquid crystals, such as lamellar phases, under polarized light. It is well known that lamellar dispersions containing large multilamellar vesicles show domains with a characteristic Maltese cross pattern. ${ }^{32}$ Moreover, by use of DIC lenses which enhance contrast between object and background and allow a resolution down to $0.5 \mu \mathrm{m}$, this technique is optimal to detect giant vesicles under normal light. $^{33}$

Cryo-Transmission Electron Microscopy. Cryo-TEM is a particularly suitable technique for the direct visualization of surfactant aggregates ranging in size from about 5-10 $\mathrm{nm}$ to 1 $\mu \mathrm{m}$. The samples were prepared according to the usual procedure, described as follows. The sample is placed in the controlled environment vitrification chamber at room temperature ${ }^{34}$ where the relative humidity is kept close to saturation to prevent water evaporation from the sample. A $5 \mu \mathrm{L}$ drop of the solution is put on a carbon-coated holey film ${ }^{35}$ supported by a TEM copper grid. After the drop is gently blotted with filter paper in order to create a thin liquid film over the grid, it is rapidly plunged into liquid ethane at its melting temperature, so that a vitrified film is obtained. The vitrified specimen is then transferred under a liquid nitrogen environment by use of a cold stage unit (model 626, Gatan Inc., Warrendale, PA) into the electron microscope, a JEOL 2000FX operating at $100 \mathrm{kV}$ with a nominal underfocus of $4 \mu \mathrm{m}$. The working temperature is kept below $-168{ }^{\circ} \mathrm{C}$, and the images are recorded on Kodak SO-163 film.

NMR Self-Diffusion. The self-diffusion coefficients of water in the isotropic solutions were determined at $25^{\circ} \mathrm{C}$ by the pulsed field gradient spin-echo (PGSE) NMR technique, ${ }^{36,37}$ by use of two different spin-echo pulse sequences.

Hahn Spin-Echo. This is the basic pulse sequence for NMR self-diffusion (Figure 1a), in which two rf pulses are used: a $90^{\circ}$ pulse at time $t=0$ and a $180^{\circ}$ pulse at time $t=\tau$, giving rise to an echo signal at $t=2 \tau$. In addition, two field gradient pulses with time duration $\delta$ and separation $\Delta$ between their leading edges are placed on either side of the second rf pulse. The first gradient pulse labels the spins by their Larmor frequency, while the second one completely refocuses only those which have not changed their positions during the time $\Delta$. At time $2 \tau$, the spin vectors are again in phase and a spin-echo occurs, with lower intensity due to translational diffusion. The echo intensity at time $2 \tau$ for Gaussian diffusion is given by the Stejskal-Tanner equation ${ }^{36,37}$

$$
I(2 \tau)=I_{0} \mathrm{e}^{-2 \tau / T_{2}} \mathrm{e}^{-\gamma^{2} g^{2} \delta^{2}(\Delta-\delta / 3) D}
$$

where $I_{0}$ is the echo intensity for $\delta=0, T_{2}$ is the transverse relaxation time, $\gamma$ is the proton magnetogyric ratio, $g$ is the magnitude of the gradient pulse, $D$ is the self-diffusion coefficient and $\tau, \delta$, and $\Delta$ are as defined above. Since the times $\tau$ and $T_{2}$ are constant, eq 1 can be simplified to

$$
I(k)=I_{0}^{\prime} \mathrm{e}^{-k D}
$$

where $k=(\gamma \delta g)^{2}(\Delta-\delta / 3)$. Thus $D$ can be readily determined 


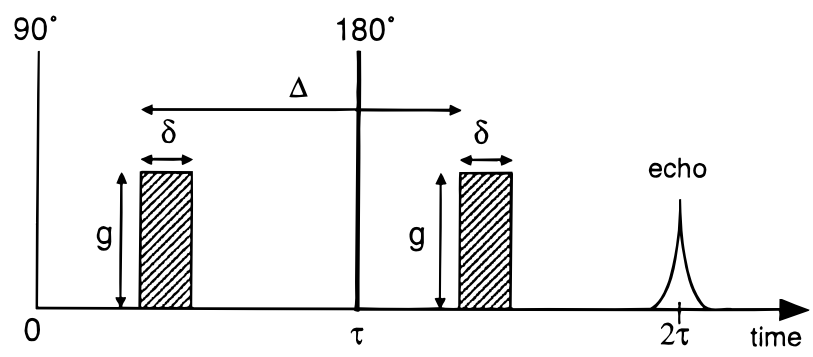

b

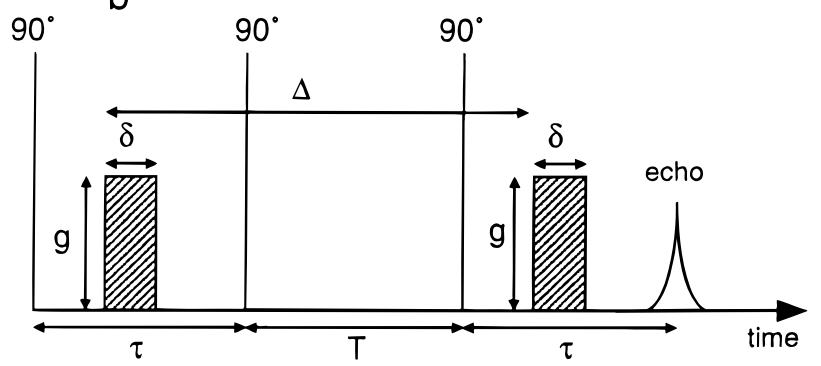

Figure 1. Spin-echo sequences used in the pulsed field gradient NMR method: (a) Hahn echo; (b) stimulated echo.

by measuring the intensity decay of a Fourier transform starting at the center of the echo (echo attenuation) as a function of $g$, $\delta$, or $\Delta$ (typically $\delta=$ is varied, at constant gradient strength $g$ and time $\Delta$ ).

A key point in the PGSE diffusion experiment is that the transport of a molecule is measured over the time span $\Delta$, which can be varied in the range of $1 \mathrm{~ms}$ to a few seconds. $\Delta$ is thus the time scale of the experiment, and if the molecules experience some sort of boundary with respect to their diffusion during $\Delta$, the outcome of the experiment is substantially changed. ${ }^{38}$ The mean square displacement of the molecule measured during $\Delta$ is given by $\left\langle z^{2}\right\rangle=2 D \Delta$, where $D$ is the molecular self-diffusion coefficient. For the case of water in our samples, with typical values of $D$ of $(1-2) \times 10^{-9} \mathrm{~m}^{2} \mathrm{~s}^{-1}$ and $\Delta=0.1 \mathrm{~s}$, the rms displacement measured is of the order of $10-20 \mu \mathrm{m}$.

A Surrey Medical Imaging Systems Inc. (England) NMR spectrometer interfaced to a JEOL FX-100 magnet equipped with an external ${ }^{2} \mathrm{H}$ lock was used. The gradient unit box was of "in-house" construction. Temperature in the probe was 25 $\pm 0.3{ }^{\circ} \mathrm{C}$, controlled with a JEOL NM 5471 variable-temperature control unit. $\Delta$ was chosen as 70 or $140 \mathrm{~ms}$, and the pulse length $\delta$ varied between 3 and $60 \mathrm{~ms}$, at a constant gradient strength of $0.01 \mathrm{~T} / \mathrm{m}$. Samples were left in the probe for 15 min for thermal equilibration before measurement. The water self-diffusion was measured in SDS-rich solutions. For many samples, a complex echo decay was obtained, composed of a fast- and a slow-diffusing component of very low molar fraction. Only the initial part of the slow-component decay could be measured even at the highest gradient available $(0.1 \mathrm{~T} / \mathrm{m})$. This component was assigned to water entrapped inside the vesicles within the experimental time scale (see discussion below). To further investigate the dynamics of water exchange, the stimulated spin-echo technique was used.

Stimulated Spin-Echo. For systems containing spins with short transverse relaxation time $T_{2}$ and a longitudinal relaxation time $T_{1}$ larger than $T_{2}$, the stimulated echo is favorably used to measure self-diffusion coefficients. Surfactant molecules in vesicle bilayers and in emulsion films, for example, have short $T_{2}$ values. Since both the slow-diffusing component of water and the surfactant diffusion (this to be included in a coming report) were measured simultaneously in vesicle solutions, it was convenient to use the stimulated echo. Yet, this sequence was not strictly necessary for the monitoring of the water slowdiffusing component, since a signal arising from the water could be obtained with the Hahn echo. The stimulated echo sequence, where the influence of $T_{2}$ relaxation in the spin-echo is minimized, consists of three $90^{\circ} \mathrm{rf}$ pulses (Figure 1b). The field gradient pulses are switched on before the second and after the third rf pulse. The echo attenuation is given by

$$
I(\tau, T)=\frac{1}{2} I_{0} \mathrm{e}^{-T / T_{1}} \mathrm{e}^{-2 \tau / T_{2}} \mathrm{e}^{-\gamma^{2} g^{2} \delta^{2}(\Delta-\delta / 3)}
$$

where $\tau$ is the time between the first two $90^{\circ}$ pulses, $T$ is the time between the second and the third $90^{\circ}$ pulse, and the other parameters are as defined before. A Bruker DMX 200 spectrometer with a gradient probe providing a maximum of $8.8 \mathrm{~T} / \mathrm{m}$ was used. The water ${ }^{1} \mathrm{H}$ echo intensity was followed as a function of the gradient strength $g$ while $\delta$ was kept constant.

\section{Diffusion of Water in a Solution of Vesicles}

The diffusion of water in a solution of surfactant aggregates is subject to obstruction (excluded volume) and, in the case of vesicles, also to confinement, due to the existence of the vesicle membrane separating enclosed water from bulk water. Detailed considerations on water diffusion in vesicles have been presented recently; ${ }^{39}$ a concise view relevant to this work follows. The outcome of a diffusion experiment for water in a solution of vesicles is dependent on the time it takes for a molecule to travel the vesicle diameter, $\tau_{\text {diff }}$, i.e., the time at which the water rms displacement equals the vesicle radius; the bilayer permeability coefficient, $P$, which sets an average residence time for water inside the vesicle, $\tau_{\text {res }}$; and the experimental observation time, $\Delta$ in the PGSE diffusion experiment. In most vesicle systems, it is observed that $\tau_{\text {diff }} \ll \tau_{\text {res }}$, i.e., the vesicle membrane constitutes a barrier for free water diffusion. The water molecules bounce several times against the vesicle inner wall before they leave the vesicle. Then a situation of fast or slow exchange of water between the interior and the exterior of the vesicle may be detected, depending on the chosen time scale $\Delta$.

(i) Fast Exchange Case ( $\left.\Delta>\tau_{\text {res }}\right)$. In this case only a single, population-weighted averaged diffusion coefficient is obtained. A two-site model, similar to the one for free and bound water in a micellar system, can be applied ${ }^{38}$

$$
D_{\mathrm{obs}}=p_{\mathrm{ves}} D_{\mathrm{ves}}+\left(1-p_{\mathrm{ves}}\right) A D_{0}
$$

where $D_{\text {obs }}$ is the measured water diffusion coefficient, $D_{\text {ves }}$ is the diffusion coefficient for the water inside the vesicle which equals that of the vesicle for the case of relatively small unilamellar vesicles (since the measured rms displacement of water is much larger than the vesicle size), $D_{0}$ is the diffusion coefficient for the bulk water, $p_{\text {ves }}$ is the fraction of water inside the vesicles, and $A$ is an obstruction factor (accounting for excluded volume effects). Assuming the vesicles to be monodisperse hard spheres, $A$ takes the form of ${ }^{40}$

$$
A=1 /\left(1+\Phi_{\mathrm{ves}} / 2\right)
$$

where $\Phi_{\text {ves }}$ is the volume fraction of vesicles. If the first term of eq 4 is neglected, since $p_{\text {ves }} D_{\text {ves }} \ll D_{0}$, and we further identify $p_{\mathrm{ves}} \approx \Phi_{\mathrm{ves}}$, assuming that the vesicle radius is much larger 
than the bilayer thickness, eq 4 can be rearranged to

$$
\Phi_{\mathrm{ves}}=2\left(1-D / D_{0}\right) /\left(2+D / D_{0}\right)
$$

with the vesicle radius $R_{\text {ves }}$ obtained by

$$
R_{\mathrm{ves}}=6 \Phi_{\mathrm{ves}} /\left(N_{\mathrm{A}} C_{\mathrm{t}} a_{\mathrm{s}}\right)
$$

where $N_{\mathrm{A}}$ is Avogadro's number, $C_{\mathrm{t}}$ is the total surfactant concentration, and $a_{\mathrm{s}}$ is the average surfactant headgroup area.

(ii) Slow exchange case $\left(\boldsymbol{\Delta}<\boldsymbol{\tau}_{\text {res }}\right)$ in a monodisperse system. In this case there is a fast-diffusing component for the water outside the vesicles experiencing obstruction and a slowdiffusing component for the water inside experiencing entrapment. For monodisperse vesicles, assuming that the $T_{2}$ values are the same for the water inside and outside the vesicles (reasonable if the vesicles are not too small), the echo attenuation is a biexponential function of $k$ :

$$
I(k) / I_{0}=\left(1-p_{\text {ves }}\right) \mathrm{e}^{-k D_{\text {out }}}+p_{\text {ves }} \mathrm{e}^{-k D_{\text {ves }}}
$$

where $D_{\text {out }}$ and $D_{\text {ves }}$ are the diffusion coefficients for the water outside and inside the vesicles, respectively. The vesicle radius is obtained from $D_{\text {out }}$ by noticing that $D_{\text {out }}=A D_{0}$. Moreover, the enclosed water must be diffusing with the vesicle, i.e., its diffusion coefficient equals that of the vesicle (see discussion above), which is several orders of magnitude smaller than $D_{\text {out }}$. Hence, in a slow exchange case, the fast and slow decays are well separated and from a plot of $\log \left(I / I_{0}\right)$ vs $k$ one can obtain $\Phi_{\text {ves }}$ by extrapolating the slow decay to $k=0$. The hydrodynamic radius of the vesicle can then be also directly obtained from the Stokes-Einstein equation accounting for excluded volume and hydrodynamic effects. ${ }^{39}$

(iii) Intermediate Case and Polydispersity Effects. The cases described before can be considered as the two extremes. As a consequence, there is a certain time scale for which the exchange dynamics is intermediate between slow and fast. The intermediate case shows up in a semilog plot of the echo decay vs $k$, when $\Delta$ is increased, as a departure from two straight lines (slow case), with the appearance of a gradual curvature until one straight line is obtained (fast case). In any vesicle solution there is always size polydispersity. Therefore, there is a range of residence times for the water inside the vesicles, since the residence time $\tau_{\text {res }}$ is proportional to the vesicle radius through ${ }^{9}$

$$
\tau_{\mathrm{ves}}=R_{\mathrm{ves}} / 3 P
$$

where $P$ is the bilayer permeability coefficient for water. Assuming $P$ on the order of $10^{-4}-10^{-3} \mathrm{~cm} \mathrm{~s}^{-1}$, as measured for fluid lecithin bilayers, ${ }^{9}$ a residence time of about $10 \mathrm{~ms}$ is obtained for a 100-nm-radius vesicle, while a time of about 1 $\mathrm{s}$ is obtained for a $10 \mu \mathrm{m}$ vesicle. Hence, a complex water echo decay may arise due to the simultaneous effect of size polydispersity and the three possible cases of exchange for a given size and a given time scale described above. If there is slow water exchange for all polydisperse vesicles in solution, at a certain time scale, then the echo decay of the slow component is not a mono- but a multiexponential function of $k{ }^{39}$ An appropriate distribution function of $D$ has to be chosen to fit the data, such as the Kohlrausch-Williams-Watts type distribution or, more meaningfully, the log-normal distribution. ${ }^{38}$ The latter can then be converted, in principle, to a distribution function of particle radius. However, it may happen that a situation of all-in-slow or all-in-fast exchange is never detected for the vesicles due to high polydispersity and the limited range of $\Delta$ available. Depending on vesicle size, water may be in fast exchange in some vesicles, in slow exchange in others, and in the intermediate case for the remaining vesicles. Then the curvature of the decay of the slow-diffusing component is $\Delta$-dependent in a complex way, and any fitting procedure becomes difficult. Another source of complication arises from the fact that for very large vesicles (diameter $>1 \mu \mathrm{m}$ ) the rms displacement of a water molecule measured for typical $\Delta$ values is of the same magnitude as the vesicle diameter, in which case the molecule experiences restricted diffusion. ${ }^{38}$ This situation will also contribute to the profile of the echo-decay of the slowdiffusing component.

\section{Results and Discussion}

1. General View of the Phase Behavior. The phase behavior for the catanionic mixture SDS-DDAB-water over the entire concentration range was reported previously at 40 ${ }^{\circ} \mathrm{C} .{ }^{4}$ The temperature used was a few degrees above the Krafft boundary of the system. Several liquid crystalline phases, namely, cubic and lamellar, were found in complex phase equilibria (Figure 2a). In this work, the phase behavior in the very dilute region was investigated in detail, at $25^{\circ} \mathrm{C}$ since the Krafft boundary was found to depend only on those of the individual surfactants $\left(17^{\circ} \mathrm{C}\right.$ for SDS and $20{ }^{\circ} \mathrm{C}$ for DDAB). The surfactant concentration is presented in weight percent (for a maximum of 3 wt $\%$ in total surfactant) or, when convenient, in total surfactant molar concentration, $C_{\mathrm{t}}=C_{\mathrm{SDS}}+C_{\mathrm{DDAB}}$. The mixing ratio is given as the molar fraction of DDAB in the surfactant mixture, $X_{\mathrm{DDAB}}=C_{\mathrm{DDAB}} /\left(C_{\mathrm{SDS}}+C_{\mathrm{DDAB}}\right)$.

As shown in Figure $2 b$, both solution and liquid crystalline phases appear in the phase diagram of this mixture. At equimolarity, crystals of the complex $\mathrm{DDA}^{+} \mathrm{DS}^{-}$(catanionic surfactant) form in a clear solution which contains the remaining $\mathrm{NaBr}$. Appropriate heterogeneous regions were also experimentally found in both the SDS-rich and the DDAB-rich side, bearing in mind that since catanionic mixtures are in reality four-component systems, multiphase regions can only be approximately depicted in triangular phase diagrams. ${ }^{3,41}$ In the cationic-rich side, an isotropic bluish turbid solution was found, consisting of a narrow lobe up to about 0.8 wt $\%$ DDAB (Figure $2 b$ ). At higher surfactant concentration, the phase behavior is dominated by the DDAB-rich swollen lamellar phase and the catanionic crystals in appropriate two- and three-phase regions. A detailed study of the phase behavior and microstructure in the DDAB-rich side will be reported later. ${ }^{42}$

The phase behavior in the SDS-rich area, depicted in the phase diagrams of Figures $2 \mathrm{~b}$ and $3 \mathrm{a}$, will now be described in detail. It is the result of combined data from direct imaging methods, such as electron and light microscopy, and complementary methods such as NMR water self-diffusion, ocular inspections, and turbidity measurements.

1.1. Single-Phase Region (Solution). SDS forms a micellar solution in water with a cmc of $0.24 \mathrm{wt} \%$. A solution is still present on addition of DDAB but only up to $X_{\mathrm{DDAB}}=0.06$, which defines a solubilization boundary at this composition (Figure 2b). The solution is colorless for an SDS concentration higher than $1 \mathrm{wt} \%$, but slightly bluish below this value. For $X_{\mathrm{DDAB}}>0.06$, samples with more than 0.5 wt $\%$ SDS display a two-phase region, consisting of a precipitate-like material and a bluish solution. However, between 0.5 and 1.2 wt \% SDS, the precipitate-like material dissolves at a certain $X_{\mathrm{DDAB}}$ and a bluish solution reforms. For less than $0.5 \mathrm{wt} \%$ SDS, as DDAB is added, a single-phase solution region is always present up to the appearance of the catanionic crystals at $X_{\mathrm{DDAB}}=0.34$ 


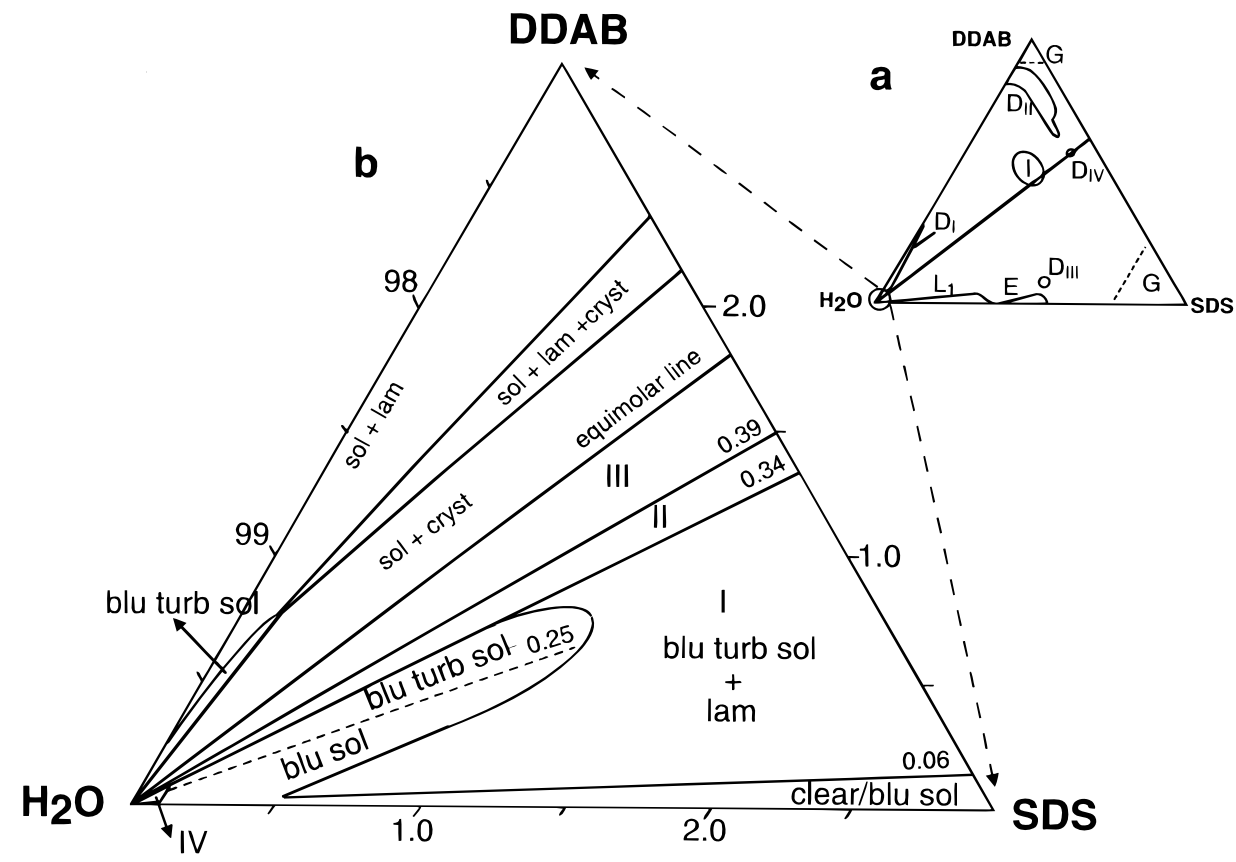

Figure 2. Phase diagrams for the SDS-DDAB-water system: (a) Over the entire concentration range at $40{ }^{\circ} \mathrm{C}$, as reported previously; ${ }^{4}$ phase notations are $\mathrm{L}_{1}$, isotropic micellar; E, normal hexagonal; I, cubic; $\mathrm{D}_{\mathrm{I}-\mathrm{IV}}$, lamellar; and G, crystalline phases; (b) at $25^{\circ} \mathrm{C}$ in the water-rich corner (maximum surfactant concentration 3 wt \%); abreviations are blu, bluish; turb, turbid; sol, solution; lam, lamellar phase; and cryst, crystals. See Figure 3 for the description of multiphase regions I-IV.

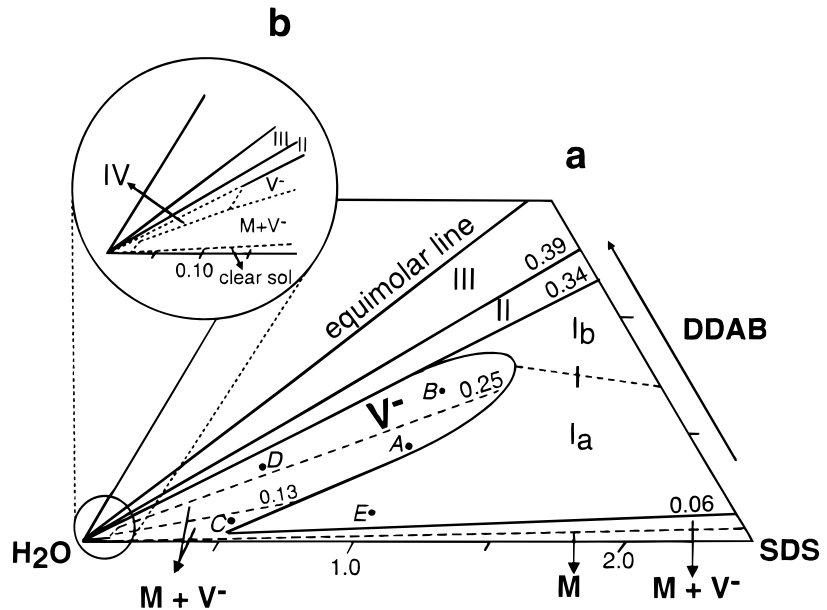

Figure 3. Phase microstructure in the SDS-rich side of the SDS$\mathrm{DDAB}-$ water system at $25^{\circ} \mathrm{C}$. (a) Solution regions of different microstructure are $\mathrm{M}$, spheroidal micelles; $\mathrm{M}+\mathrm{V}^{-}$, coexisting micelles (spheroidal, below $X_{\mathrm{DDAB}}=0.13$ and disklike, above) and vesicles; and $\mathrm{V}^{-}$, vesicles. Points $\mathrm{A}-\mathrm{E}$ give the composition of the samples shown in Figures 4A-E. Multiphase regions are I, solution and lamellar phase of high (subregion $I_{a}$ ) or low (subregion $I_{b}$ ) density; II, vesicle solution, lamellar phase and catanionic solid; and III, catanionic solid and vesicle solution. Numbers are the values of $X_{\mathrm{DDAB}}$ for relevant phase (or region) boundaries. (b) Expanded view of water apex, where multiphase IV is a mixture of solution and catanionic solid.

(Figure 2b). Turbidity measurements made in this region, at constant $C_{\mathrm{t}}$ and increasing molar fraction of added DDAB (00.34 ), showed a marked increase in turbidity at ca. $X_{\mathrm{DDAB}}=$ 0.25 (data not shown). Even to the naked eye, solutions change from moderately to fairly bluish and turbid around this value. Hence, a broken line was drawn in Figure $2 \mathrm{~b}$ at $X_{\mathrm{DDAB}}=0.25$ to indicate this observation.

1.2. Multiphase Regions. A two-phase region is present above the solubilization boundary (I in Figure $2 b$ ), consisting of a bluish solution in which a cloudy phase separates either at the bottom (as mentioned above) or at the top. Light microscopy gave evidence for the lamellar liquid-crystalline nature of this phase (cf. Section 4). In Figure 3a, it can be seen that region I is actually subdivided into $I_{a}$, where the lamellar phase is of higher density than the solution, and $I_{b}$, where the opposite occurs. It is likely that this lamellar phase is the same as the one occurring at much higher SDS concentration, $\mathrm{D}_{\mathrm{III}}$ in Figure $2 \mathrm{a}^{4}$ Region II is a narrow three-phase region $\left(X_{\mathrm{DDAB}}=0.34-\right.$ $0.39)$, containing a bluish solution in which mixed lamellar domains/catanionic crystals separate at the top (cf. Section 4). Samples in this region phase-separate rather slowly (hours to days). On the other hand, for $X_{\mathrm{DDAB}}=0.39-0.50$, the $\mathrm{DDA}^{+} \mathrm{DS}^{-}$catanionic crystals immediately precipitate out from a colorless solution (region III). A significant observation was the detection of region IV, seen in detail in Figure 3b. It defines as a solution in which the catanionic solid appears with time, either as dispersed flocs or, for more dilute samples, as a white precipitate. This region sets a stability limit for the solution phase. If a solution with $X_{\mathrm{DDAB}}=0-0.06$ is continuously diluted, no detectable precipitation occurs, whereas if $X_{\mathrm{DDAB}}>$ 0.06 , a precipitate always appears at some point in dilution. The solution/precipitate boundary was not investigated in detail, but it was observed that for $X_{\mathrm{DDAB}}=0.13-0.20$, precipitation occurs at $0.025 \mathrm{wt} \% \mathrm{SDS}$, whereas for $X_{\mathrm{DDAB}}=0.25-0.29$, it occurs at $0.10 \mathrm{wt} \% \mathrm{SDS}$. Thus, as $X_{\mathrm{DDAB}}$ is made higher, the solution phase becomes less stable than the catanionic solid.

2. Investigation of Solution Microstructure by CryoTEM. The microstructural characterization of the SDS-rich solution was done by direct imaging using the cryo-TEM technique. Both dilution paths and paths with a variable mixing ratio at a fixed total surfactant concentration were investigated. The microstructural characterization was based on many micrographs per sample. From these, five representative micrographs (Figure 4A-E) were selected, corresponding to points $\mathrm{A}-\mathrm{E}$ in Figure $3 \mathrm{a}$. On the basis of the cryo-TEM study, boundaries were drawn inside the solution phase, separating regions of different microstructure: $\mathrm{M}$, micellar region; $\mathrm{V}^{-}$, vesicle region; and $\mathrm{M}+\mathrm{V}^{-}$, region of coexisting micelles and vesicles (subdivided into two regions, as shown later). 


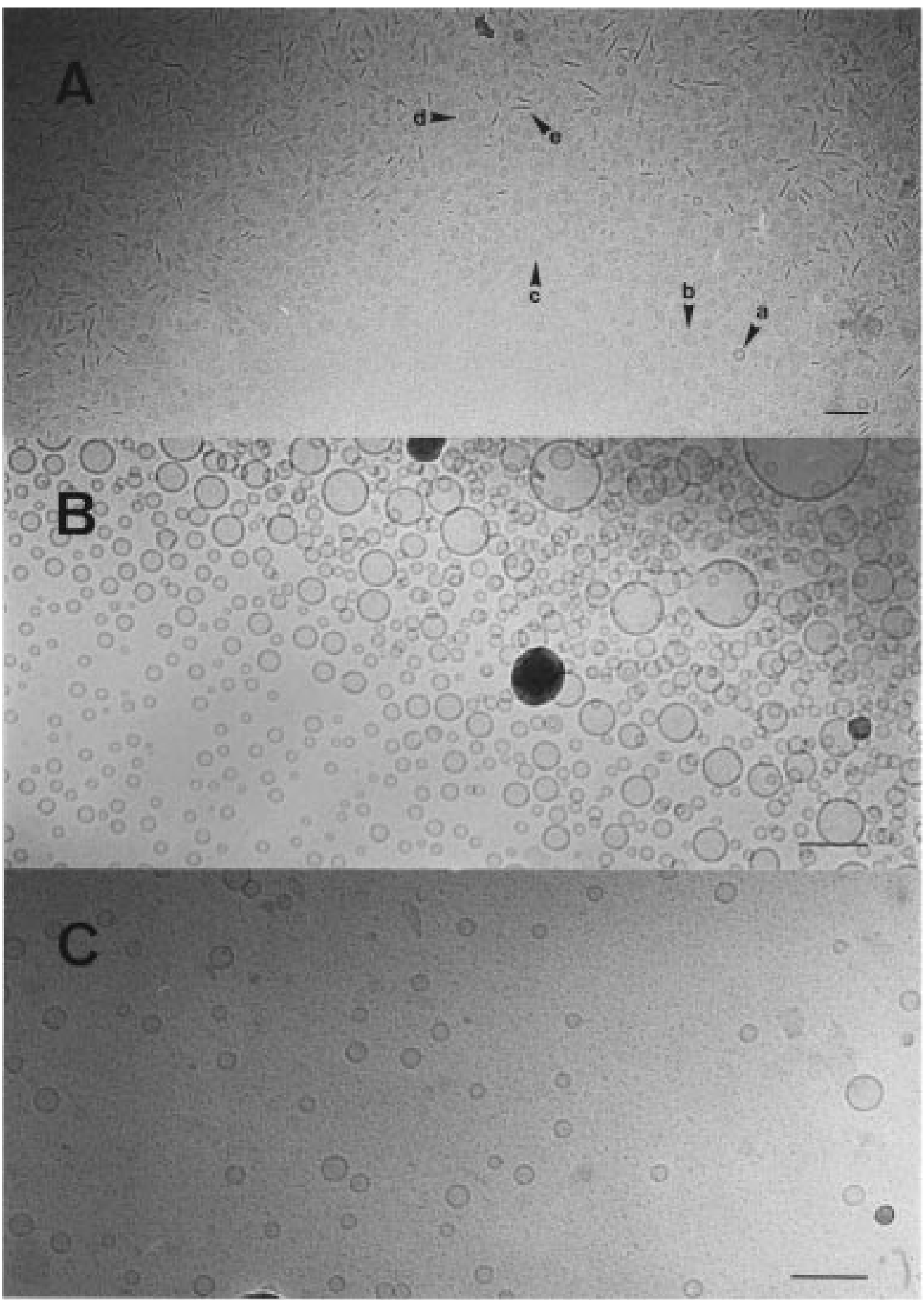

Figure 4. Microstructure of SDS-rich solutions in the SDS-DDAB-water system as obtained by Cryo-TEM: (A) (1.0 wt $\%$ SDS, $\left.X_{\mathrm{DDAB}}=0.20\right)$, small unilamellar vesicles, either spherical (a) or nonspherical (b), in coexistence with disklike micelles, seen in face-on (c and d) and edge-on (e) projections; (B) (1.0 wt \% SDS, $\left.X_{\mathrm{DDAB}}=0.30\right)$, unilamellar vesicles; (C) $\left(0.5 \mathrm{wt} \% \mathrm{SDS}, X_{\mathrm{DDAB}}=0.11\right)$, spheroidal micelles in coexistence with small unilamellar vesicles; (D) $\left(0.5 \mathrm{wt} \%\right.$ SDS, $\left.X_{\mathrm{DDAB}}=0.30\right)$, unilamellar vesicles; (E) $\left(1 \mathrm{wt} \% \mathrm{SDS}, X_{\mathrm{DDAB}}=0.09\right)$, clusters of large bilayer fragments (lamellar phase) coexisting with micelles and small unilamellar vesicles (multiphase region $\mathrm{I}_{\mathrm{a}}$ ); Bar, $100 \mathrm{~nm}$.

2.1. $M$ and $M+V^{-}$Region. Samples lying in the $\mathrm{M}+\mathrm{V}^{-}$ region were found to contain micelles in coexistence with small unilamellar vesicles of diameter between 15 and $40 \mathrm{~nm}$. The size and shape of the micelles as well as the volume fraction of vesicles are dependent on the mixing ratio between the two surfactants.

Figure $4 \mathrm{~A}$, for $1 \mathrm{wt} \%$ SDS and $X_{\mathrm{DDAB}}=0.20$ (A in Figure 3a), shows the coexistence of two main types of aggregates: (i) small unilamellar vesicles and (ii) disklike aggregates. For a suitable distinction between these aggregates, it is to be realized that a TEM micrograph is a $2 \mathrm{D}$ projection of a $3 \mathrm{D}$ reality, since the depth of focus is much larger than the sample thickness. Hence, a disklike aggregate is clearly distinguishable from a vesicle in TEM: while the vesicle displays an edge contour due to mass thickness contrast, the disk in face-on position with respect to the electron beam has uniform contrast from center to edge and thus no contour. In Figure 4A, the vesicles appear as spherical aggregates with a diameter of 20- 


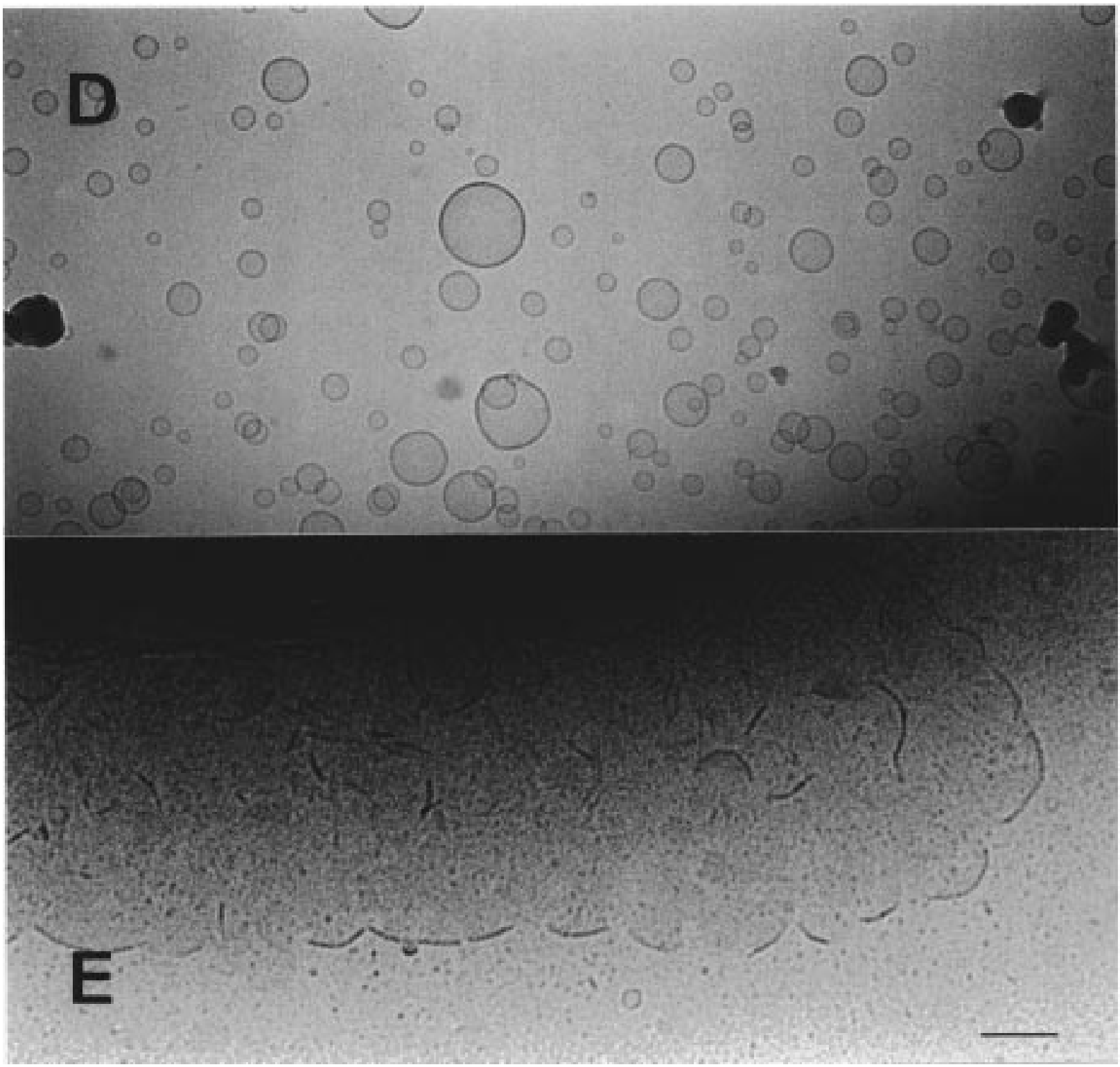

Figure 4. Cont.

$30 \mathrm{~nm}$, showing a clear edge contour (a) or as nonspherical structures, displaying a low-contrast contour (b). In turn, clear evidence for the presence of disks comes from the fact that both face-on projections without contour (c and d) and edge-on projections (e) are visible. These projections result from the different orientation of the disks with respect to the electron beam. The face-on projection is either quasicircular (c) or in most cases elliptical (d), suggesting that the disks are anisotropic in shape. The edge-on projections (e) have a length in the range of 30-70 nm. Such disklike aggregates were reproduced for the same composition 2 months after the initial imaging. As mentioned below, similar aggregates were found for other compositions in the phase diagram. On the basis of these observations, they are regarded as disklike micelles. One can speculate that their stability may derive from an accumulation of the single-chained SDS in the highly curved rim and a more even distribution of both surfactants in the flat part.

Spheroidal micelles are nevertheless the dominant structure in the $\mathrm{M}+\mathrm{V}^{-}$region for $X_{\mathrm{DDAB}}<0.06$, i.e., below the solubilization boundary. A detailed investigation of samples with $0.40-0.50$ wt $\%$ SDS and increasing $X_{\text {DDAB }}$ was carried out. Since for these compositions a bluish solution was always present up to solid formation, a micelle-to-vesicle transition could be continuously followed. Spheroidal micelles coexist with vesicles for $X_{\mathrm{DDAB}}=0.03-0.12$, and they constitute the dominant agreggate form. This can be observed in Figure 4C, at $X_{\mathrm{DDAB}}=0.11$, where spheroidal micelles not larger than 5-6 $\mathrm{nm}$ in diameter are visible together with small unilamellar vesicles. For $X_{\mathrm{DDAB}}=0.13-0.22$, however, the spheroidal micelles are replaced with disklike micelles. These disks are mostly seen in the edge-on orientation with an average length of $50 \mathrm{~nm}$, similar to those in Figure 4A (at higher SDS concentration). Another significant observation, based on many micrographs, is that as $X_{\mathrm{DDAB}}$ increases within the full range of the solution region, an increasing number of vesicles of about the same size range is visible, indicating that the volume fraction of vesicles is gradually increasing.

The influence of dilution in the solution microstructure was also investigated, for two dilution paths. At $X_{\mathrm{DDAB}}=0.20$, when the SDS concentration decreases from 1 to $0.2 \mathrm{wt} \%$, disklike micelles are still present in coexistence with vesicles. Hence, dilution does not seem to induce microstructural change for the range of mixing ratios where disks occur. On the basis of this observation, a dashed line was drawn inside the $\mathrm{M}+\mathrm{V}^{-}$region for constant $X_{\mathrm{DDAB}}=0.13$, dividing it into two subregions (Figure 3a): below the line, spheroidal micelles coexist with 
vesicles and above it, only disklike micelles and vesicles are present. For a lower $X_{\mathrm{DDAB}}$ value, however, a dilution path results in different effects. Samples at fixed $X_{\mathrm{DDAB}}=0.06$ ranging from 10 to $0.1 \mathrm{wt} \%$ SDS were imaged (data not shown). At $10 \mathrm{wt} \%$ only spheroidal micelles and no other type of aggregates are present. Between 1 and $0.1 \mathrm{wt} \%$, spheroidal micelles and small vesicles are always observed, but with dilution the fraction of vesicles increases significantly, at the expense of micelles. At 0.1 wt \% SDS, practically only vesicles were seen, some of which with larger size (100-200 nm). Dynamic light scattering results reported before ${ }^{4}$ also indicated large aggregates in solution $(260 \mathrm{~nm})$ at less than $0.1 \mathrm{wt} \%$ SDS and roughly the same mixing ratio. It is thus clear that dilution at this mixing ratio induces (i) a change from a micellar solution to a solution of coexisting micelles and vesicles and (ii) an increase in the fraction of vesicles and in their average size.

A qualitative explanation for these dilution effects might be found in the cmc reduction effects typically observed in catanionic mixtures. $^{3}$ As the molar fraction of the minority surfactant is increased, the $\mathrm{cmc}$ of the mixture gradually decreases, reaching a minimum at the equimolar ratio. At a low amount of added cationic surfactant as $X_{\mathrm{DDAB}}=0.06$, the decrease in the cmc of SDS is probably not significant. Therefore, upon dilution below the $\mathrm{cmc}$ value, an increasing fraction of SDS is in monomeric form, the aggregates are increasingly enriched in $\mathrm{DDAB}$, and their shape and size change (electrostatic and packing effects). At $X_{\mathrm{DDAB}}=0.20$, on the other hand, dilution within the solution region does not change the monomeric surfactant concentration which is expected to be very low (due to significant cmc reduction); thus no significant structural changes take place in the solution.

A small number of vesicles was detected by cryo-TEM for such a low value of $X_{\mathrm{DDAB}}$ as 0.03 , at $0.5 \mathrm{wt} \% \mathrm{SDS}$. The SDS-rich solution thus contains large aggregates already with a very small addition of DDAB. It is likely that the packing constraints imposed by DDAB on the mixed surfactant monolayer are so strong that it is more favorable for the system to evolve into a mixture of aggregates of large difference in curvature (micelles and vesicles) than for the micelles to grow indefinitely (e.g., to long rods or threadlike micelles). Nevertheless, there must be a region in the phase diagram where only micelles are present (region $\mathrm{M}$ ); hence, a lower boundary for $\mathrm{M}+\mathrm{V}^{-}$was drawn, and the $\mathrm{M}$ region was defined in Figure $3 a$.

Cryo-TEM evidence for the two-phase region $I_{a}$ described before was also obtained and is shown in Figure $4 \mathrm{E}$, for $X_{\mathrm{DDAB}}$ $=0.09$ and $1.0 \mathrm{wt} \%$ SDS. Spheroidal micelles and small unilamellar vesicles in the range of $20-100 \mathrm{~nm}$ are seen (solution phase). In addition, large bilayer fragments organized in clusters of more than $0.5 \mu \mathrm{m}$ appear, indicating the presence of a lamellar phase.

2.2. $V^{-}$Region. The boundaries for the vesicle region $\mathrm{V}^{-}$ $\left(X_{\mathrm{DDAB}}=0.25\right.$ and 0.34 , Figure $\left.3 \mathrm{a}\right)$ were defined on the basis of cryo-TEM results. Bluish solutions within that composition range contain only vesicles (Figures 4B and 4D). As mentioned before, turbidity measurements gave further evidence for the lower boundary of $\mathrm{V}^{-}$, since a significant increase in turbidity was measured around $X_{\mathrm{DDAB}}=0.25$. In general, practically only unilamellar vesicles with a size distribution falling in the range of 20-200 nm were imaged; the size range is often biased toward a narrower width, 20-70 nm (Figures 4C and 4D).

The effect of increasing $X_{\mathrm{DDAB}}$ on the vesicle size and polydispersity was investigated, at fixed total surfactant concentration $C_{\mathrm{t}}=20 \mathrm{mM}$ (around $0.5 \mathrm{wt} \% \mathrm{SDS}$ ). Inside the $\mathrm{M}+\mathrm{V}^{-}$region, the average vesicle size appears to remain

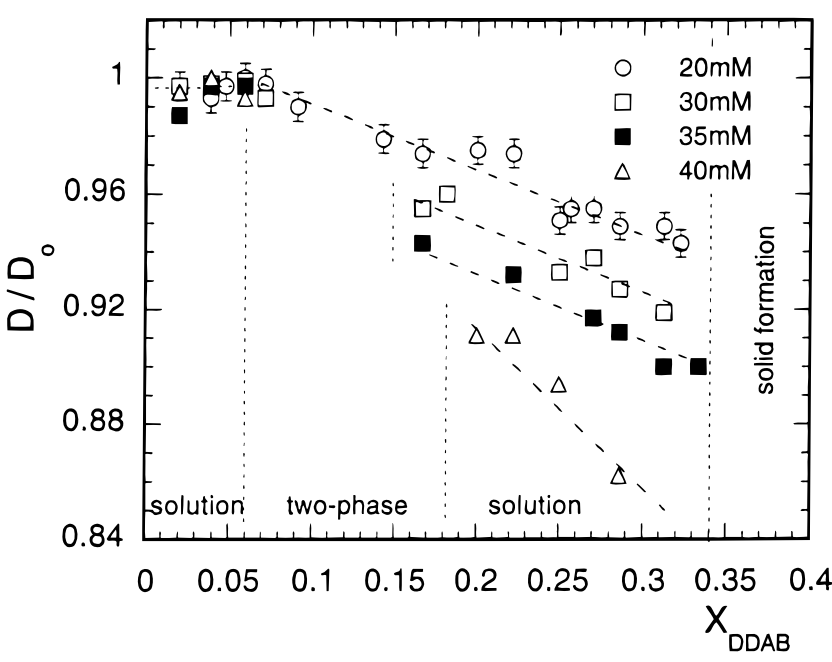

Figure 5. Reduced water self-diffusion coefficient as a function of molar fraction of added DDAB, at constant total surfactant concentration, $C_{\mathrm{t}}=C_{\mathrm{SDS}}+C_{\mathrm{DDAB}}$. For $C_{\mathrm{t}}=20 \mathrm{mM}$, a continuous solution region exists; for $C_{\mathrm{t}}=30-40 \mathrm{mM}$, a two-phase region separates the solutions (cf. phase diagrams and text). Lines are guides to the eye.

constant at about $30 \mathrm{~nm}$ with increasing $X_{\mathrm{DDAB}}$; also, a narrow size distribution $(20-40 \mathrm{~nm})$ is seen throughout the region. As $X_{\mathrm{DDAB}}$ approaches the lower $\mathrm{V}^{-}$boundary at 0.22 , the polydispersity increases, with the formation of larger aggregates (100$200 \mathrm{~nm}$ ) and bilamellar vesicles (vesicles with two concentric bilayers). As $X_{\mathrm{DDAB}}$ increases from 0.25 to 0.31 within the vesicle region, the average vesicle size also increases from 30 to $50 \mathrm{~nm}$; simultaneously, the size distribution becomes wider (from $20-100 \mathrm{~nm}$ to $20-250 \mathrm{~nm}$ ). The increase in aggregate size can be intuitively understood if one considers that as $X_{\mathrm{DDAB}}$ increases, more of a double-chained cationic surfactant is incorporated into a negatively charged vesicle; consequently, the bilayer mean curvature decreases. In turn, an explanation for the increase in polydispersity with increasing $X_{\mathrm{DDAB}}$ does not seem so straightforward.

Dilution within the vesicle region does not seem to have any significant effect on the average size and size distributions of vesicles. This is exemplified by Figures $4 \mathrm{~B}$ and 4D, where there is only a decrease in concentration of similarly sized vesicles on going from $4 \mathrm{~B}$ to $4 \mathrm{D}$. Consequently, a dilution path simply induces a gradual reduction of the volume fraction of vesicles by a decrease in concentration of aggregates with essentially constant size.

Finally, it should be noted that cryo-TEM is suitable for the imaging of colloidal particles in the range of 5-1000 nm, but any particles larger than this are excluded from the vitrified sample. Thus, the characterization of size distributions solely on the basis of this method may be biased toward that particular size range.

3. Water Self-Diffusion Measurements. Water self-diffusion measurements were done in order to obtain (i) further information on the evolution of aggregate structure with DDAB addition and (ii) an estimation of vesicle sizes which would allow us to complement the cryo-TEM data.

Figure 5 shows the reduced diffusion coefficient of water (the observed diffusion coefficient, $D$, over the value for neat water, $D_{0}$, at the same temperature) as a function of $X_{\mathrm{DDAB}}$, at constant total surfactant concentration $C_{\mathrm{t}}$. For $C_{\mathrm{t}}=20 \mathrm{mM}$ $\left(0.5-0.7\right.$ wt $\%$ in total surfactant), $D / D_{0}$ can be continuously monitored from $X_{\mathrm{DDAB}}=0$ until the formation of a catanionic solid, while for higher concentrations a two-phase region separates the $\mathrm{M} / \mathrm{M}+\mathrm{V}^{-}$and the $\mathrm{V}^{-}$solutions (Figure 3a). Since 


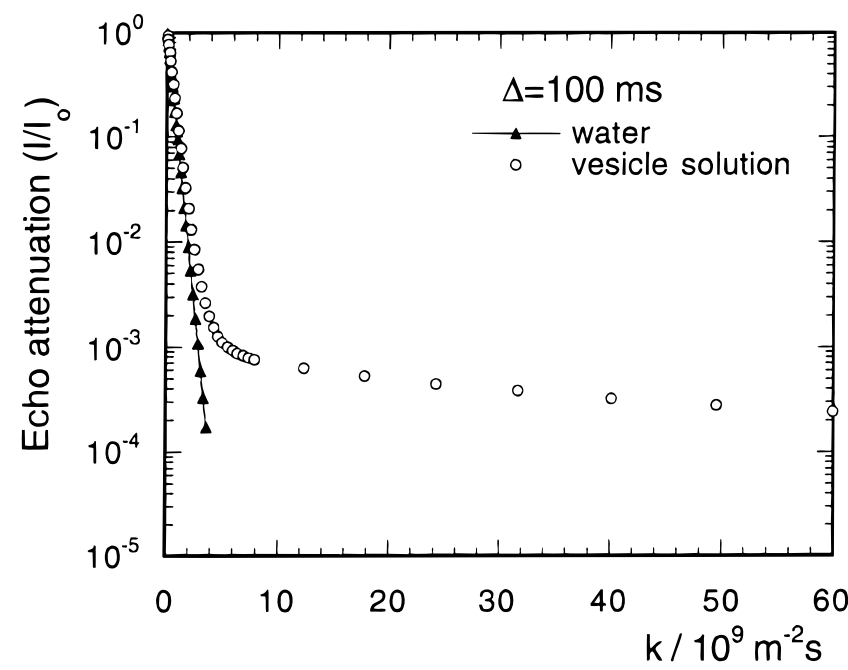

Figure 6. Echo attenuation as a function of $k=(\gamma \delta g)^{2}(\Delta-\delta / 3)$ for water in a solution of vesicles, at $1.1 \mathrm{wt} \% \mathrm{SDS}$ and $X_{\mathrm{DDAB}}=0.29$, where a slow-diffusing component of very low molar fraction is observed; the monoexponential decay for neat water (linear fit) is also shown for comparison.

the surfactant concentration is kept constant, changes in $D / D_{0}$ reflect changes in solution microstructure (changes in size, shape, or number of aggregates). As seen in Figure 5, up to $X_{\mathrm{DDAB}}=0.06, D / D_{0}$ is virtually constant and equal to unity for all concentrations. This implies that any aggregates present are causing nondetectable obstruction to water diffusion; the solution contains mainly micellar aggregates which at low concentration cause minimal obstruction effects. At about $X_{\mathrm{DDAB}}=0.07$, there is a break in the curve for $20 \mathrm{mM}$ and $D / D_{0}$ decreases gradually until solid formation. In the current method, this break signals the detection of vesicles causing entrapment and obstruction effects to water diffusion (cf. eq 4). As $X_{\mathrm{DDAB}}$ increases, the volume fraction of vesicles increases at the expense of micelles, hence the observed decrease in the reduced diffusion coefficient. In the region of only vesicles, $X_{\mathrm{DDAB}}=0.25-0.34$, the small decrease in $D / D_{0}$ can be explained by vesicle growth. This decrease is most evident at the highest concentration, since water diffusion is more sensitive to obstruction and entrapment at high surfactant concentrations. Overall, these results are consistent with the cryo-TEM data. They imply a continuous transition from micelles to vesicles as DDAB is added to an SDS solution, followed by a moderate vesicle growth in the vesicle region.

In Figure 6, a plot of the echo attenuation $\left(\log I / I_{0}\right)$ vs $k$ is shown for water in a vesicle solution $\left(1.1 \mathrm{wt} \% \mathrm{SDS}, X_{\mathrm{DDAB}}=\right.$ 0.29 ), at a typical value of $\Delta=100 \mathrm{~ms}$. The echo decay for neat water is also presented. As is evident, the echo decay in the vesicle solution is nonexponential; there is a fast- and a slow-diffusing component. When the slow decay is extrapolated to $k=0$, the obtained molar fraction is about 0.001 . So despite the fact that practically all water spins are in fast exchange between the vesicle outside/inside, there is a slow component originating from vesicles in slow or intermediate exchange (section III). These vesicles cannot be those in the range of 20-70 $\mathrm{nm}$ imaged by cryo-TEM, since the volume fraction of enclosed water for this particular solution is much higher than 0.001 (by about 2 orders of magnitude). Moreover, the residence time of water in $20-70 \mathrm{~nm}$ vesicles is shorter than the experimental time scale of $\Delta=100 \mathrm{~ms}$ used (cf. eq 9).

The plot in Figure 6 is representative of the echo decays obtained for samples in the $\mathrm{V}^{-}$region. Since from the discussion above a situation of fast exchange is present in all vesicles except for a negligible fraction (less than 0.001 in

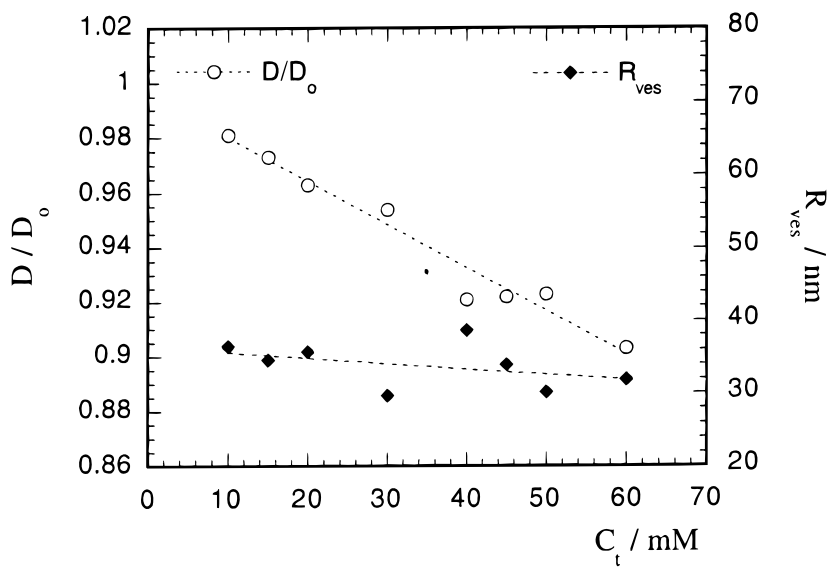

Figure 7. Estimation of vesicle sizes $(\diamond)$ from the water reduced selfdiffusion coefficients $(O)$ obtained for samples with constant $X_{\mathrm{DDAB}}=$ 0.29 along a dilution path, $C_{\mathrm{t}}=10-60 \mathrm{mM}$, where $C_{\mathrm{t}}=C_{\mathrm{SDS}}+C_{\mathrm{DDAB}}$ (lines are guides to the eye).

volume fraction), an estimation of sizes along a dilution path can be obtained. In Figure 7 , the $D / D_{0}$ values for samples at fixed $X_{\mathrm{DDAB}}=0.29$ (at midrange inside $\mathrm{V}^{-}$) are plotted vs $C_{\mathrm{t}}$ $=10-60 \mathrm{mM}$. The vesicle size was obtained from eq 7 , with two major assumptions. First, vesicle monodispersity was assumed, which obviously does not correspond to the real case. However, the obtained $D$ value is averaged over the entire population of vesicles in fast exchange so the extraction of a mean size has physical meaning. Second, a value of $a_{\mathrm{s}}=35$ $\AA^{2}$ in eq 7 was used, and this is justified as follows. The mixing ratio in the sample is $C_{\mathrm{SDS}} / C_{\mathrm{DDAB}}=2.5$, and this is taken as the vesicle composition (no other aggregates are present and the SDS monomer concentration is negligible). Due to electrostatic attractions between the surfactant headgroups, a good estimation of the average $a_{\mathrm{s}}$ value is given by the vesicle composition and the minimal cross sectional area of a hydrocarbon chain in a fluid state, ca. $25 \AA^{2}$. A simple calculation yields $a_{\mathrm{s}} \approx 35 \AA^{2}$. From the data in Figure 7, several conclusions can be drawn. First, the vesicle sizes obtained are on the order of 30-35 $\mathrm{nm}$ and thus in reasonable agreement with the cryo-TEM data. Second, the vesicle size is practically independent of concentration, which indicates that along a dilution path the volume fraction of vesicles decreases by keeping the size constant, also in agreement with cryo-TEM observations.

The origin of the slow component in the echo decays was investigated for the solution in Figure 6 by varying the observation time $\Delta$. In Figure 8 a $\log -\log$ plots of the echodecays for $\Delta=10-1000 \mathrm{~ms}$ are shown, for better visualization of the slow-component decay. As $\Delta$ increases, the fraction of the slow component gradually decreases. For the $\Delta$ range used, the decrease is of about 2 orders of magnitude, from an already low initial molar fraction of 0.001. Attempts to fit the whole curve with a biexponential function were unsuccessful, which indicates a distribution of diffusion coefficients for the slow component. No meaningful fit for the slow decay was obtained either with a Kohlrausch-Williams-Watts distribution or a lognormal distribution function; also apparent in Figure $8 \mathrm{a}$ is the $\Delta$-dependence of the profile of the slow decay. Altogether, these facts point out to an exchange dynamics described in section III(iii), that is, size polydispersity and different regimes of exchange for the vesicles act simultaneously to create a complex decay. The effect of sonication on the profile of the echo decay for the same solution further clarifies this point. In Figure $8 \mathrm{~b}$ the influence of sonication time on the echo decay is shown for fixed $\Delta=20 \mathrm{~ms}$. The cavitation induced by 

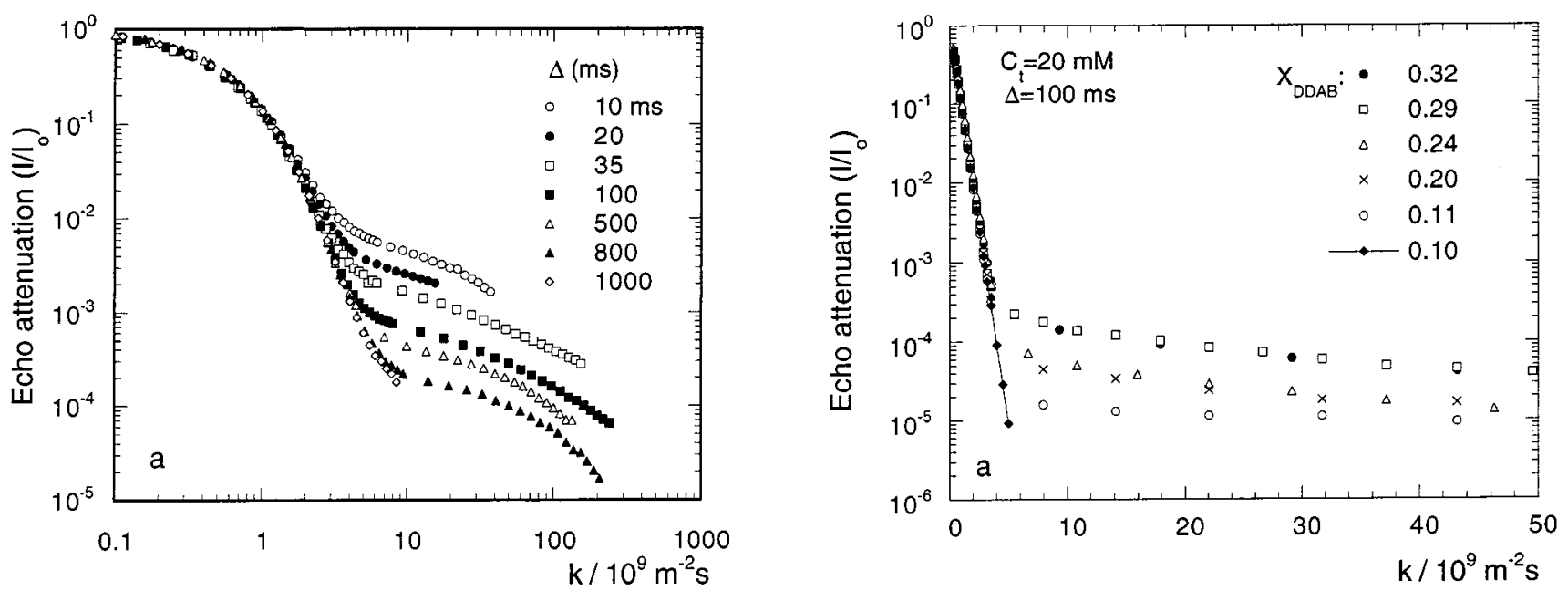

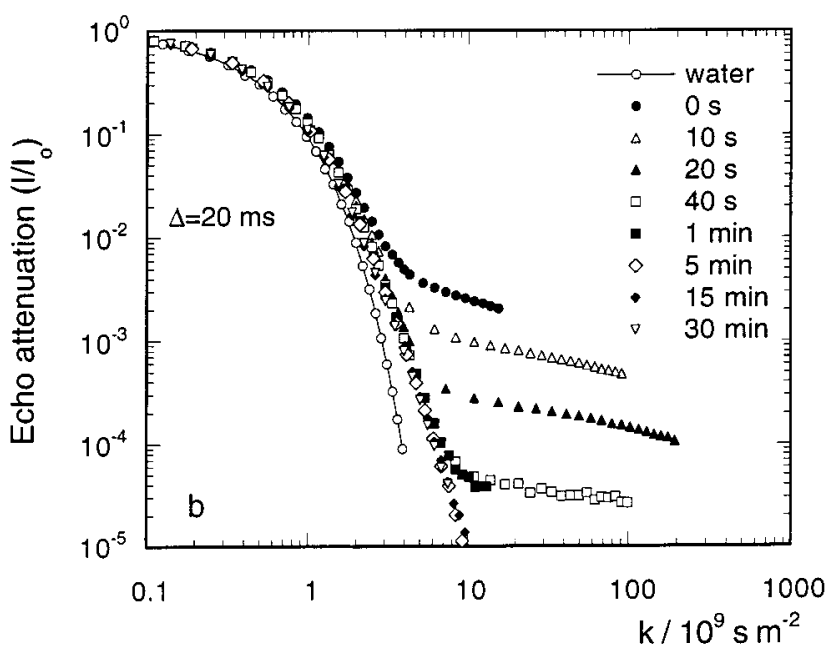

Figure 8. $\log -\log$ plot of the echo decay of water as a function of $k$ $=(\gamma \delta g)^{2}(\Delta-\delta / 3)$ for a vesicle sample with $1.1 \mathrm{wt} \%$ SDS and $X_{\mathrm{DDAB}}$ $=0.29$ : (a) for increasing values of the experimental time scale $\Delta$ (ms) and (b) at fixed $\Delta=20 \mathrm{~ms}$, for samples treated by sonication with increasing time ( $0 \mathrm{~s}$ to $30 \mathrm{~min}$ ); the decay for neat water is also shown (linear fit).

ultrasonication in a polydisperse vesicle solution/dispersion breaks any large aggregates (giant vesicles or onions) into bilayer fragments that reseal to form small unilamellar vesicles. The longer the sample is treated, the more the size distribution is shifted to lower sizes and the narrower it becomes. As can be seen in Figure 8b, by increasing the sonication time from $10 \mathrm{~s}$ to $1 \mathrm{~min}$, the fraction of slow component decreases drastically (ca. 2 orders of magnitude). For a period of sonication of $5 \mathrm{~min}$ and more, the slow component is absent.

The results in Figures $8 \mathrm{a}$ and $8 \mathrm{~b}$ can be interpreted if one considers that there is a broad distribution of very large vesicles in solution and that these vesicles have a very small volume fraction. As the observation time $\Delta$ is increased, some of these vesicles change from a regime of slow $\left(\Delta<\tau_{\text {res }}\right)$ to fast $(\Delta>$ $\tau_{\text {res }}$ ) exchange and consequently the volume fraction of water enclosed by them no longer contributes to the magnitude of the slow component. As the sonication time increases, at fixed observation time, the larger vesicles in slow exchange are increasingly destroyed into smaller ones at fast exchange and consequently the fraction of slow component rapidly decreases. The fact that the slow component could still be detected (though only marginally) at $\Delta=1000 \mathrm{~ms}$ (Figure 8a) implies that vesicles larger than $10 \mu \mathrm{m}$ are present, according to the dependence of residence time on radius. The existence of such

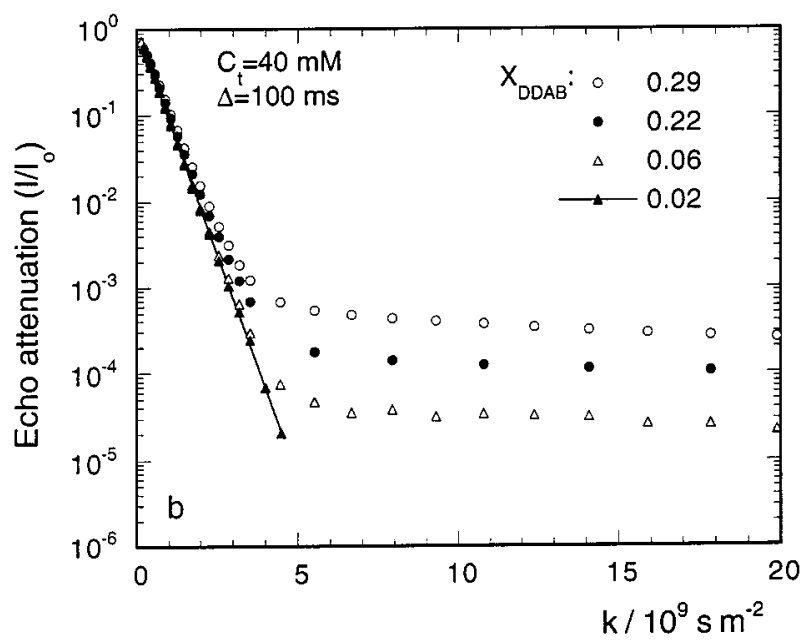

Figure 9. Semilog plot of the echo decay for water as a function of $k=(\gamma \delta g)^{2}(\Delta-\delta / 3)$ for samples with constant total surfactant concentration, $C_{\mathrm{t}}=C_{\mathrm{SDS}}+C_{\mathrm{DDAB}}$, and increasing molar fraction of added DDAB, $X_{\mathrm{DDAB}}$ : (a) $C_{\mathrm{t}}=20 \mathrm{mM}$ and (b) $C_{\mathrm{t}}=40 \mathrm{mM}$. Linear fits to the echo decay for $X_{\mathrm{DDAB}}=0.10$ in (a) and $X_{\mathrm{DDAB}}=0.02$ in (b) are shown.

giant vesicles in low concentration was indeed confirmed by light microscopy, as shown below.

The occurrence of any water slow-diffusing component for samples in the $\mathrm{M}+\mathrm{V}^{-}$region was also investigated. In Figures $9 \mathrm{a}$ and $9 \mathrm{~b}$ the echo decays are plotted for different values of $X_{\mathrm{DDAB}}$, at fixed concentration $C_{\mathrm{t}}=20$ and $40 \mathrm{mM}$, respectively. At $20 \mathrm{mM}$ the slow component is detected only for $X_{\mathrm{DDAB}}>$ 0.10 ; below this value, only a monoexponential decay is obtained (straight line). At $40 \mathrm{mM}$, the slow component is traced at $X_{\mathrm{DDAB}}=0.06$, corresponding to the solubilization boundary in the phase diagram; the samples below this value show only a monoexponential decay (straight line). In both cases, the fraction of slow component clearly increases as the amount of added DDAB increases and it is highest for compositions in the $\mathrm{V}^{-}$region. These results clearly indicate that giant vesicles are already present at low amounts of added DDAB and not just in the vesicular region $\mathrm{V}^{-}$. Moreover, as $X_{\mathrm{DDAB}}$ increases, an increasing fraction of giant vesicles is present.

4. Light Microscopy Observations. Representative samples were inspected in a light microscope equipped with DIC lenses to check for the presence of the large vesicles detected by the water self-diffusion. At $1.1 \mathrm{wt} \% \mathrm{SDS}$ and $X_{\mathrm{DDAB}}=0.29$ (corresponding to $C_{\mathrm{t}}=55 \mathrm{mM}$ ), aggregates larger than $0.8 \mu \mathrm{m}$ 


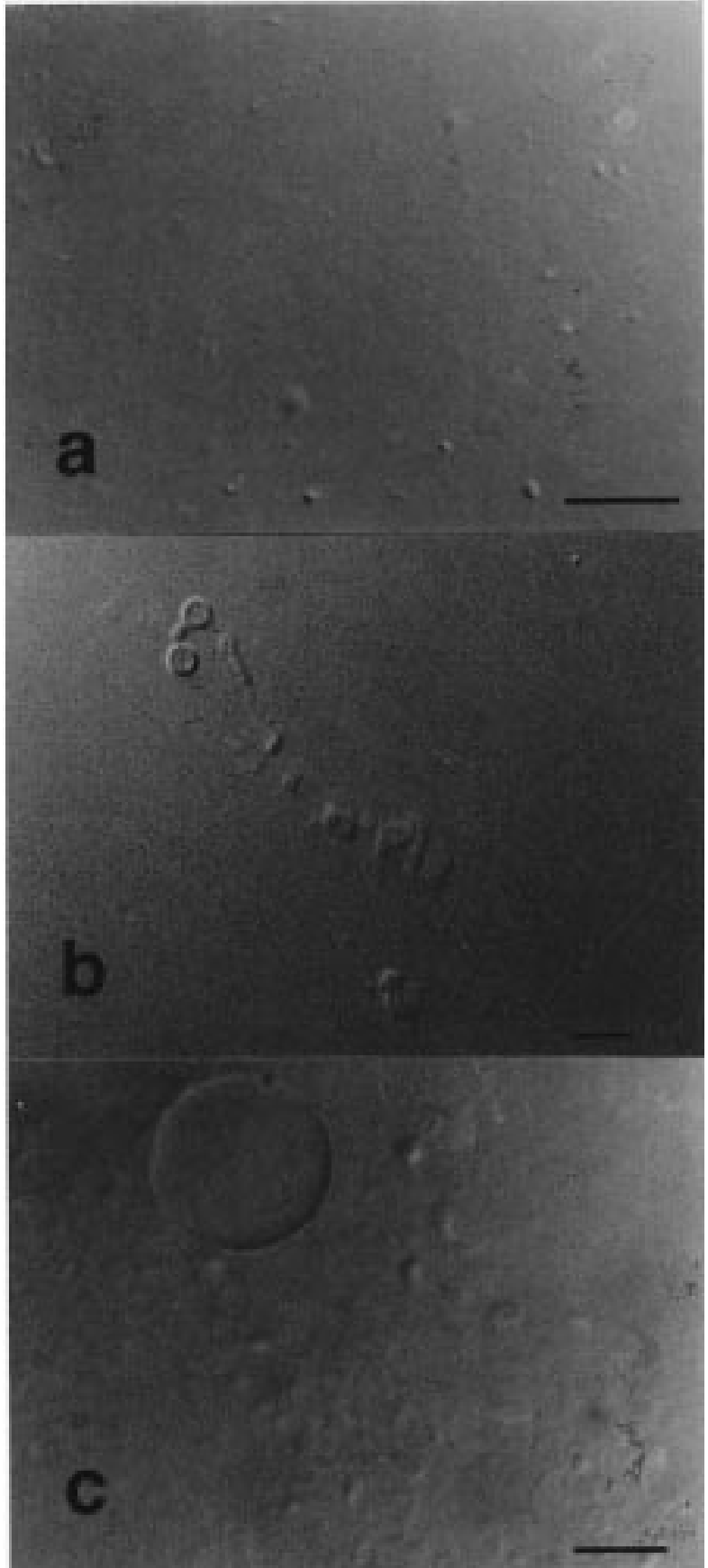

Figure 10. Light micrographs of a turbid bluish solution in the SDS/ DDAB mixture $\left(1.1 \mathrm{wt} \%\right.$ SDS, $\left.X_{\mathrm{DDAB}}=0.29\right)$ : (a) Mainly very large vesicles with $0.8-2.0 \mu \mathrm{m}$ diameter are seen; (b) $5-10 \mu \mathrm{m}$-sized vesicles, occasionally attached to each other, and tubular structures also present; and (c) a giant vesicle of $40 \mu \mathrm{m}$ diameter. Bars: (a) and (b), $10 \mu \mathrm{m}$; (c), $20 \mu \mathrm{m}$.

were indeed observed (Figure 10a-c). Giant vesicles of a diameter between 0.8 and $2 \mu \mathrm{m}$ are the dominant structures (Figure 10a), but a smaller fraction of even larger structures, 5-40 $\mu \mathrm{m}$ vesicles and tubules, are also seen (Figures $10 \mathrm{~b}$ and 10c). The occurrence of these giant vesicular aggregates is sparse compared with typical lamellar dispersions of similar surfactant concentration. Therefore, one concludes that the aggregates have a rather low volume fraction in the solution, in agreement with water self-diffusion data.

Samples at lower fixed concentration, $C_{\mathrm{t}}=20 \mathrm{mM}$, and with mixing ratios outside the vesicle region were also inspected. For $X_{\mathrm{DDAB}}=0.10$ and slightly higher, a small number of spherical vesicles of diameters in the range of $1-10 \mu \mathrm{m}$ could be detected. This observation is reasonably consistent with the detection by PGSE NMR of a slow-diffusing component of water for $X_{\mathrm{DDAB}}=0.11$, shown in Figure 9a (for $X_{\mathrm{DDAB}}=0.10$, probably the fraction of the giant vesicles is too low to be detected by this method). At $X_{\mathrm{DDAB}}=0.29$, giant vesicular aggregates of sizes $1-40 \mu \mathrm{m}$ were observed, indicating an increase in polydispersity.

Outside the isotropic solution region, light microscopy showed that the precipitate-like material in region I consists of a lamellar phase. At lower total surfactant concentration, a mixture of flocculated multilamellar vesicles (onions) of several $\mu \mathrm{m}$ in size and tubular structures was detected. At higher concentration, a flow birefringent phase with mosaic texture was viewed under polarized light. The top flocs in multiphase region III appear as a mixture of solid crystallites and flocculated onions and tubules of $\mu \mathrm{m}$ size.

\section{Summary}

1. Sequence of Aggregate Structures upon DDAB Addition. The sequence of aggregate geometries in the SDS/DDAB mixture, as the surfactant mixing ratio is varied, can be rationalized at a qualitative level in terms of an interplay between electrostatic effects and geometric packing constraints. When the cationic double-tailed surfactant DDAB is added to a micelle of the single-tailed anionic SDS (or to an SDS-rich aggregate) two major effects are expected: a decrease in both intra- and interaggregate electrostatic repulsions, due to partial surface charge neutralization and higher concentration of counterions, and an increase in the effective surfactant packing parameter due to the incorporation of a double-chained surfactant in the amphiphilic interface. These two effects will in turn induce, concomitantly, an increase in the size of the surfactant aggregates and a decrease in the preferred curvature of the surfactant film.

The addition of DDAB to SDS micelles, however, was seen to induce initially very limited micellar growth. Only a few molecules of DDAB seem to be incorporated into a spheroidal micelle before another type of aggregate of much larger aggregation number and lower mean curvature-a vesicle-is formed. A continuous transition from the region of only micelles $\left(X_{\mathrm{DDAB}}<0.03\right)$ to that of only vesicles $\left(0.25<X_{\mathrm{DDAB}}\right.$ $<0.34$ ) is thus present, involving a wide region of coexistence of the two types of aggregates. This type of continuous, nonfirst-order micelle-to-vesicle transition has been observed in other catanionic systems. ${ }^{12,15,16,19,23}$ It contrasts with first-order transitions reported in other mixtures, ${ }^{13}$ which involve a macroscopic phase separation between micellar and vesicle solutions. In the SDS/DDAB mixture, a structural change occurs in any case to the micelles which coexist with vesicles; at some critical ratio of added DDAB (ca. $X_{\mathrm{DDAB}}=0.13$ ), the spheroidal micelles are no longer stable and disklike micelles were found to exist.

Discoidal micelles have been reported previously in very few mixed surfactant systems on the basis of cryo-TEM imaging, in mixtures of double-chained lipids with asymmetric chain length ${ }^{43}$ and, recently, in the SDS-lecithin system. ${ }^{44}$ They were also believed to be intermediate structures in lecithin-bile salt systems, ${ }^{45}$ but recent studies clearly indicate cylindrical polymerlike micelles to be present instead. ${ }^{46}$ Formation of disklike micelles as an intermediate geometry in micelle-to-vesicle transitions thus seems to be relatively uncommon. The change in aggregate geometry during the solubilization of phospholipid bilayers by surfactants has been recently theoretically mod- 
eled. ${ }^{47,48}$ Kozlov et al. employed a curvature energy descripition of the surfactant monolayer to propose a simple criterion for disk formation, requiring that the ratio between the Gaussian curvature modulus $\bar{\kappa}$ and the bending rigidity $\kappa$ be less than $-0.2{ }^{48}$ Although such relatively large magnitudes of $\bar{\kappa}$ are not common for uncharged amphiphiles, in ionic systems the extra electrostatic contribution to these elastic moduli can be sizable. Indeed, both diverge, in the ratio $\bar{\kappa} / \kappa \approx 3$, in the limits of low electrolyte and low volume fraction of amphiphile, ${ }^{49}$ allowing the Kozlov criterion to be met from the conditions in the mixed SDS/DDAB system. Further investigation of this issue requires a structural study of the system in the presence of added salt.

2. Vesicle Size and Stability. For a narrow range of surfactant mixing ratios, $\mathrm{SDS} / \mathrm{DDAB} \approx 3-2: 1$ in molar ratio, only vesicles and no other aggregate structures were found to be stable. Combined microscopy and water self-diffusion data imply that the vesicle size distribution is extremely wide, i.e., there is a high polydispersity in the system. In principle, the diffusion data in Figures $8 \mathrm{a}$ and $8 \mathrm{~b}$ are not incompatible with a very broad, continuous size distribution from the smaller 20$100 \mathrm{~nm}$ vesicles detected by cryo-TEM to the $1-50 \mu \mathrm{m}$-sized ones traced by light microscopy. Yet, the detailed cryo-TEM study did not indicate the presence of any aggregates in the size range of $200-800 \mathrm{~nm}$. If they were present, even in low concentration (from self-diffusion data), they would have been easily visualized, as shown in other systems. ${ }^{19,31}$ All the data thus strongly points to a bimodal type of distribution, much biased toward the smallest vesicles $(20-100 \mathrm{~nm})$ and with a very small volume fraction of giant vesicles $(1-50 \mu \mathrm{m})$. It remains to be investigated whether this distribution corresponds to the true equilibrium size distribution. We note that a Poisson-Bolztmann cell model recently proposed ${ }^{50}$ accounts for equilibrium two-population distributions, comprising small and large unilamellar vesicles, in ionic-nonionic surfactant mixtures.

Since the $\mathrm{V}^{-}$lobe extends only to a maximum of $1.2 \mathrm{wt} \%$ $\operatorname{SDS}\left(C_{\mathrm{t}} \approx 60 \mathrm{mM}\right)$, the maximal volume fraction of vesicles is also relatively low (approximately 0.10 ), certainly far from a regime of vesicle close packing. This suggests that the stability of the vesicle phase is essentially dictated by electrostatic (longrange) interactions between aggregates, highly sensitive to the screening effect of the released counterions. Finally, we point out that the SDS-rich vesicles found in this system have several important features. They are obtained spontaneously, in the sense that no preparation method other than simple mixing of individual surfactant solutions is required (e.g. no highly energetic method). The vesicles are considerably stable with time; after 2 years of shelf storage no signs of phase separation are visible. They are in equilibrium with other phases, namely, lamellar phases and catanionic solids, i.e., heterogeneous samples are found where either of these is one of the constituent phases. Altogether, these observations strongly suggest that these vesicles are a thermodynamically stable phase. A more stringent criteria for the definition of such an equilibrium system, though, is the nondependence of size distribution on the preparation method and the full reversibility of any formation path. These important kinetic and equilibrium aspects are currently under investigation.

Acknowledgment. We thank O. Söderman and M. Nydén for interesting and valuable discussions on water self-diffusion results. U. Olsson and H. D. Burrows are also acknowledged for discussions. E.F.M. is grateful to JNICT and Praxis XXI for a research studentship (BD/9295/96) and for financial support within the project 2/2.1/QUI/411.94. O.R. acknowledges a grant from the Swedish Institute for his stay in Lund. The Swedish Research Council for Engineering Sciences (TFR) is also kindly acknowledged for financial support.

\section{References and Notes}

(1) Mixed surfactant systems; Holland, P. M., Rubingh, D. N., Eds.; American Chemical Society: Washington, DC, 1992.

(2) Mixed Surfactant Systems; Ogino, K., Masahiko, A., Eds.; Marcell Dekker: New York, 1993

(3) Khan, A.; Marques, E. Catanionic Surfactants. In Specialists Surfactants; Robb, I. D., Ed.; Blackie Academic and Professional, an imprint of Chapman \& Hall: London, 1997; pp 37-76.

(4) Marques, E.; Khan, A.; Miguel, M. G.; Lindman, B. J. Phys. Chem. 1993, 97, 4729 .

(5) Caria, A.; Khan, A. Langmuir 1996, 12, 6282.

(6) Edlund, H.; Sadaghiani, A.; Khan, A. Langmuir 1997, 13, 4953

(7) Kaler, E. W.; Murthy, A. K.; Rodriguez, B. E.; Zasdzinski, J. A. N. Science 1989, 245, 1371.

(8) Ostro, M. J. Liposomes: from Biophysics to Therapy; Marcel Dekker: New York, 1987.

(9) Lasic, D. D. Liposomes: from Physics to Applications; Elsevier: Amsterdam, 1993

(10) Machluf, M.; Regev, O.; Peled, Y.; Kost, J.; Cohen, S. J. Controlled Release 1996, 43, 35 .

(11) Hargreaves, W. R.; Deamer, D. W. Biochemistry 1978, 17, 2804

(12) Kaler, E. W.; Herrington, K. L.; Murthy, A. K.; Zasadzinski, J. A J. Phys. Chem. 1992, 96, 6698

(13) Herrington, K. L.; Kaler, E. W.; Miller, D. D.; Zasadzinski, J. A.; Chiruvolu, S. J. Phys. Chem. 1993, 97, 13792.

(14) Yatcilla, M. T.; Herrington, K. L.; Brasher, L. L.; Kaler, E. W. J. Phys. Chem. 1996, 100, 5874.

(15) Ambühl, M.; Bangerter, F.; Luisi, P. L.; Skrabal, P.; Watzke, H. J. Langmuir 1993, 9, 36.

(16) Watzke, H. J. Prog. Colloid Polym. Sci. 1993, 93, 15.

(17) Lin, Z.; He, M.; Scriven, L. E.; Davis, H. T.; Snow, S. A. J. Phys. Chem. 1993, 97, 3571 .

(18) Huang, J.-B.; Zhao, G.-X. Colloid Polym. Sci. 1995, 273, 156

(19) Regev, O.; Khan, A. J. Colloid Interface Sci. 1996, 182, 95.

(20) Safran, S. A.; Pincus, P. A.; Andelman, D. A. Science 1990, 248 , 354.

(21) Kumaran, V. J. Chem. Phys. 1994, 99, 5490.

(22) Bergström, M. Langmuir 1996, 12, 2454.

(23) Söderman, O.; Herrington, K. L.; Kaler, E. W.; Miller, D. D. Langmuir 1997, 13, 5531.

(24) Kondo, Y.; Uchiyama, H.; Yoshino, N.; Nihyiama, K.; Abe, M. Langmuir 1995, 11, 2380.

(25) Israelachvili, J. N. Intermolecular and Surface Forces, 2nd ed.; Academic Press: San Diego, 1992.

(26) Warr, G. G.; Sen, R.; Evans, D. F.; Trend, J. E. J. Phys. Chem. 1988, 92,774

(27) Fontell, K.; Ceglie, A.; Lindman, B.; Ninham, B. Acta Chem. Scand. 1986, $A 40,247$

(28) Zemb, T.; Gazeau, D.; Dubois, M.; Gulik-Krzywicki, T. Europhys. Lett. 1993, 21, 759 .

(29) Caboi, F.; Monduzzi, M. Langmuir 1996, 12, 3548 673

(30) Dubois, M.; Gulik-Krzywicki, T.; Cabane, B. Langmuir 1993, 9,

(31) Regev, O.; Khan, A. Prog. Colloid Polym. Sci. 1994, 97, 298.

(32) Van de Pas, J. C. Tenside, Surfactants Deterg. 1991, 28, 158.

(33) Evans, D. F.; Wennerström, H. The Colloidal Domain-Where Physics, Chemistry, Biology and Technology Meet; VCH: New York, 1994.

(34) Bellare, J. R.; Davis, H. T.; Scriven, L. E.; Talmon, Y. J. Electron Microsc. Tech. 1988, 10, 87

(35) Vinson, P. K. Cryo-TEM, carbon-coated holey polymer film; The 45th Annual Meeting of the Electron Microscopy Society of America, San Francisco, 1987.

(36) Stilbs, P. Prog. Nucl. Magn. Reson. Spectrosc. 1987, 19, 1.

(37) Callaghan, P. T. Principles of Nuclear Magnetic Resonance Microscopy; Clarendon Press: Oxford, 1991.

(38) Söderman, O.; Stilbs, P. Prog. Nucl. Magn. Reson. Spectrosc. 1994, $26,445$.

(39) Olsson, U.; Nakamura, K.; Kunieda, H.; Strey, R. Langmuir 1996, 12,3045 .

(40) Jönsson, B.; Wennerström, H.; Nilsson, P.-G.; Linse, P. Colloid Polym. Sci. 1986, 264, 77.

(41) Jokela, P.; Jönsson, B.; Wennerström, H. Prog. Colloid Polym. Sci. 1985, 70,17

(42) Marques, E. F. Unpublished work.

(43) Vinson, P. K.; Belare, J. R.; Davis, H. T.; Miller, W. G.; Scriven, L. E. J. Colloid Interface Sci. 1991, 142, 74. 
(44) Silvander, M.; Karlsson, G.; Edwards, K. J. Colloid Interface Sci. 1996, $179,104$.

(45) Fromherz, P.; Röcker, C.; Rüppel, D. Faraday Discuss. Chem. Soc. 1986, 81, 39.

(46) Egelhaaf, S.; Schurtenberger, P. J. Phys. Chem. 1994, 98, 8560

(47) Fattal, D. R.; Andelman, D.; Ben-Shaul, A. Langmuir 1995, 11, 1154.
(48) Kozlov, M. M.; Lichtenberg, D.; Andelman, D. J. Phys. Chem. 1997, 101, 6600 .

(49) Daicic, J.; Fogden, A.; Carlsson, I.; Wennerström, H.; Jönsson, B. Phys. Rev. E 1996, 54, 3984.

(50) (a) Oberdisse, J. Manuscript. (b) Oberdisse, J.; Porte, G. Phys. Rev. E 1997, 56, 1965. 